\title{
The accuracy of nurse performance of the triage process in a tertiary hospital emergency department in Gauteng Province, South Africa
}

\author{
L N Goldstein, ${ }^{1}$ MB BCh, MMed, FCEM (SA), Cert Critical Care (SA); L M Morrow, ${ }^{2}$ BDiet; T A Sallie,${ }^{3}$ BHSc; K Gathoo, ${ }^{3}$ BHSc; \\ K Alli, ${ }^{3}$ BHSc; T M M Mothopeng, ${ }^{3}$ BHSc; F Samodien, ${ }^{3}$ BHSc \\ ${ }^{1}$ Division of Emergency Medicine, School of Clinical Medicine, Faculty of Health Sciences, University of the Witwatersrand, Johannesburg, \\ South Africa \\ ${ }^{2}$ Emergency Department, Helen Joseph Hospital, Johannesburg, South Africa \\ ${ }^{3}$ Department of Family Medicine, School of Clinical Medicine, Faculty of Health Sciences, University of the Witwatersrand, Johannesburg, \\ South Africa
}

Corresponding author: L N Goldstein (drg666@gmail.com)

\begin{abstract}
Background. Triage in the emergency department (ED) is necessary to prioritise management according to the severity of a patient's condition. The South African Triage Scale (SATS) is a hospital-based triage tool that has been adopted by numerous EDs countrywide. Many factors can influence the outcome of a patient's triage result, and evaluation of performance is therefore pivotal.

Objectives. To determine how often patients were allocated to the correct triage category and the extent to which they were incorrectly promoted or demoted, and to determine the main reasons for errors in a nurse-led triage system.

Methods. Triage forms from a tertiary hospital ED in Gauteng Province, South Africa, were collected over a 1-week period and reviewed retrospectively.

Results. A total of 1091 triage forms were reviewed. Triage category allocations were correct $68.3 \%$ of the time. Of the incorrect category assignments, $44.4 \%$ of patients were promoted and $55.6 \%$ demoted. Patients in the green category were most commonly promoted (29.4\%) and patients who should have been in orange were most commonly demoted (35.0\%). Trauma patients were more likely to be incorrectly promoted and non-trauma patients to be incorrectly demoted. Mistakes were mainly due to discriminator errors (57.8\%), followed by numerical miscalculations (21.5\%). The leading omitted discriminators were 'abdominal pain', 'chest pain' and 'shortness of breath'. Conclusions. Mis-triaging using the SATS can be attributed to incorrect or lack of discriminator use, numerical miscalculations and other human errors. Quality control and quality assurance measures must target training in these areas to minimise mis-triage in the ED.
\end{abstract}

S Afr Med J 2017;107(3):243-247. DOI:10.7196/SAMJ.2017.v107i3.11118

Triage in the emergency department (ED) is necessary in order to prioritise and assign relatively scarce resources to the medical needs of patients for efficient and timeous treatment according to the severity of their condition or acuity on presentation. ${ }^{[1]}$ Triage systems should ideally allow for patient care to be given within an acceptable time, thus decreasing overcrowding, increasing patient satisfaction, allowing urgent patients to receive appropriate care, and preventing non-urgent patients from receiving unnecessary treatment. ${ }^{[2]}$

In 2004, the South African Triage Scale (SATS) was developed to be used as a nurse-led, in-hospital triage tool. It has since been adopted by numerous EDs. ${ }^{[3]}$ The SATS categorises patients into different colour groups depending on the severity of their condition. This categorisation is based on vital signs that are used to constitute the Triage Early Warning Score (TEWS), as well as a discriminator list of conditions that acts as a safety net for certain time-critical conditions where patients may present with normal or near-normal vital signs. The aim is for healthcare providers to evaluate patients triaged red immediately, orange within 10 minutes of arrival, yellow within 1 hour and green within 4 hours. The discriminator may be used to upgrade a patient's triage category.

\section{Validity of the SATS}

Few studies have been done to determine the validity of the SATS with regard to over- or under-triage of patients. Over- and under- triage is determined by comparing the triage tool assessment with the ultimate outcome of the patient. Over-triage will result in unnecessarily assigning resources to a patient, and under-triage could mean potential morbidity or mortality as a result of time delays. Rosedale et al ${ }^{[4]}$ prospectively evaluated the SATS in a hospital in rural KwaZulu-Natal Province, South Africa (SA). The results showed an under-triage rate of $4.4 \%$ and an over-triage rate of $4.3 \%$. In a validation study, ${ }^{[5]}$ the SATS had an average over-triage rate of $15 \%$ and an under-triage rate of $10 \%$.

A classroom-based study by Dalwai et al. ${ }^{[3]}$ assessed the reliability and accuracy of the SATS when used by ED nursing staff in Pakistan. Their reference standard was based on the triage category assigned to case vignettes that had been evaluated by an expert panel (surrogate end-point). The study found a similar average over-triage result (15\%) and a slightly higher average under-triage result of $22 \%$. It was noted that $66 \%$ of emergency cases (red) were under-triaged, but fortunately only by one acuity level, to orange. ${ }^{[3]}$ However, the expert panel in the study were mostly based in high-income rather than low- and middleincome healthcare settings, which may have affected their opinion of the patients' acuity level, leading to a tendency to over-rate patients. The authors also suggested that nurses may tend to under-rate a patient's acuity level when only given a paper-based vignette. Both the abovementioned factors may have contributed to the high undertriage results, especially in the emergency cases. However, overall it 
was concluded that the SATS is reliable and can be used by nursing staff in Pakistan. ${ }^{[3]}$

It has come to our attention that studies assessing triage tools compare their over- and under-triage rates with the so-called 'acceptable ranges' developed by the American College of Surgeons Committee on Trauma (ACSCOT). ${ }^{[6]}$ This is not a valid comparator, however, as the ACSCOT triage guidelines were developed for prehospital (in-field) use in trauma patients only.

\section{Factors that affect the outcome of a patient's triage score}

Many factors can influence the outcome of a patient's triage result, but these have not been comprehensively explored in the literature. Triage errors are commonly ascribed to understaffing. Burstrom et al's ${ }^{[7]}$ study comparing three hospital triage systems found that a physician-led triage system resulted in improved efficiency and quality of patient care. Similarly, Molyneux et al ${ }^{[8]}$ emphasised the need for well-trained nurses who are able to triage and assist in resuscitation, as this could increase treatment efficacy.

Unfortunately, in the SA environment, human resource constraints do not readily allow for a physician-led triage system, or for the most-qualified nurses always to be available to perform triage. To compensate for this, the SATS was designed to be used by enrolled nursing assistants. High staff turnover sometimes results in expertise being lost, so training of new staff needs to occur on a regular basis. ${ }^{[5,8]}$

The SATS was introduced in 2010 in our ED in a tertiary hospital in Gauteng Province, SA. Induction training was offered to all enrolled nurses and enrolled nursing assistants in the ED, all of whom are responsible for triaging patients. Since then, monthly in-service training has been given.

Self-evaluation of our triage performance is pivotal, not only as part of quality assurance but to ensure timely institution of patient management in the ED.

\section{Objectives}

To determine how often patients were allocated to the correct triage category and the extent to which they were incorrectly promoted or demoted within a triage category, to determine the main reasons for promotion and demotion within triage categories, and to compare error rates for each category of triage in our nurse-led triage system.

\section{Methods \\ Study design and study setting}

Triage forms from a tertiary hospital ED in a metropolitan area of Gauteng, SA, were retrospectively reviewed. The ED sees approximately 65000 patients annually. Most patients have non-traumatic pathologies $(\sim 70 \%)$, the remainder being trauma-related. Paediatric and obstetric and gynaecology patients are mainly seen at the nearby sister hospital, but children needing resuscitation and women with ectopic pregnancies or in active labour sometimes present to the ED.

\section{Data collection}

Data were collected over the 1-week period 10 - 17 May 2015. As nursing shifts change every Wednesday, the data were representative of all four day and night shifts in the ED. Patients aged $<12$ years were not subject to the 2008 adult SATS guidelines and were therefore excluded from the study. An experienced ED doctor and a triage researcher formed the expert panel that delivered the consensus on the correct triage categories. Permission to conduct the study was granted by the Human Research Ethics Committee of the University of the Witwatersrand (ref. no. M150473).

\section{Results}

A total of 1091 triage forms were reviewed. Table 1 shows the overall triage performance.

There was no statistically significant difference between trauma and non-trauma patients with regard to overall correct triages performed (Fisher's exact test $p=0.23$ ); however, non-trauma patients were more likely to be incorrectly demoted (Fisher's exact test $p=0.0439$ ), with an odds ratio of 1.697 (95\% confidence interval 1.025 - 2.753) and trauma patients were more likely to be incorrectly promoted.

Table 2 shows the numbers of patients correctly triaged (darker blue), incorrectly promoted (grey) and incorrectly demoted (lighter blue), and Fig. 1 the numbers of patients who were triaged correctly and incorrectly (promoted or demoted) according to triage category.

Table 3 sets out the main reasons for errors made during triage, namely discriminator errors, numerical miscalculation and 'other'. Discriminator errors were either 'incorrect discriminator usage' (the nurse used a discriminator that was not correct or does not exist) or 'failure to record a discriminator' (no discriminator recorded where there should have been one). The numerical miscalculations were either attributable to human error (incorrect addition of the TEWS score) or to transposition error (choosing an incorrect TEWS category, which then resulted in an incorrect TEWS score). 'Other' refers to cases in which the nurse calculated the correct TEWS score and/or selected the correct discriminator, but still selected the incorrect triage category. Table 4 shows the common reasons for errors in triage and whether they ultimately resulted in a correct or incorrect overall triage result. Table 5 demonstrates the discriminators most often omitted and the effects thereof.

\section{Discussion}

We did not assess the accuracy of the SATS tool itself in this study, but rather the accuracy of its use by nurses in the ED. We therefore could not use the terms over- and under-triage, instead using promotion and demotion, respectively. To our knowledge no other studies have made use of these terms when referring to triage results. Only triage data were evaluated and not the ultimate outcome of the patients; an incorrect triage categorisation from the nurses may therefore still have resulted in the correct and timeous treatment of the patient.

Table 1. Triage performance

\begin{tabular}{llll}
\hline & Trauma, $\boldsymbol{n}(\%)$ & Non-trauma, $\boldsymbol{n}(\%)$ & Total, $\boldsymbol{N}(\%)$ \\
\hline Patients triaged & $301(29.0)$ & $737(71.0)$ & $1038(100)^{*}$ \\
Triages performed correctly & $214 / 301(71.1)$ & $495 / 737(67.2)$ & $709(68.3)$ \\
Triages performed incorrectly & $87 / 301(28.9)$ & $242 / 737(32.8)$ & $329(31.7)$ \\
$\quad$ Promoted patients & $47 / 87(54.0)$ & $99 / 242(40.9)$ & $146 / 329(44.4)$ \\
$\quad$ Demoted patients & $40 / 87(46.0)$ & $143 / 242(59.1)$ & $183 / 329(55.6)$ \\
${ }^{*} 53 / 1091$ triage forms (4.9\%) were considered to be indeterminate, i.e. the triage category could not be correctly assigned owing to insufficient information being documented.
\end{tabular}


Table 2. Triage assessment per triage category, $n$

\begin{tabular}{lllllll}
\hline & \multicolumn{5}{c}{ Study } \\
\cline { 2 - 7 } Nurse assessment & Indeterminate & Green & Yellow & Orange & Red & Total \\
\hline Indeterminate & 2 & 17 & 18 & 13 & 1 & 51 \\
Green & 0 & 243 & 71 & 14 & 0 & 328 \\
Yellow & 0 & 88 & 289 & 82 & 0 & 459 \\
Orange & 0 & 13 & 32 & 168 & 16 & 229 \\
Red & 2 & 0 & 3 & 10 & 9 & 24 \\
Total & 4 & 361 & 413 & 287 & 26 & 1091 \\
Darker blue = correctly triaged; grey = incorrectly promoted; lighter blue = incorrectly demoted. & & &
\end{tabular}

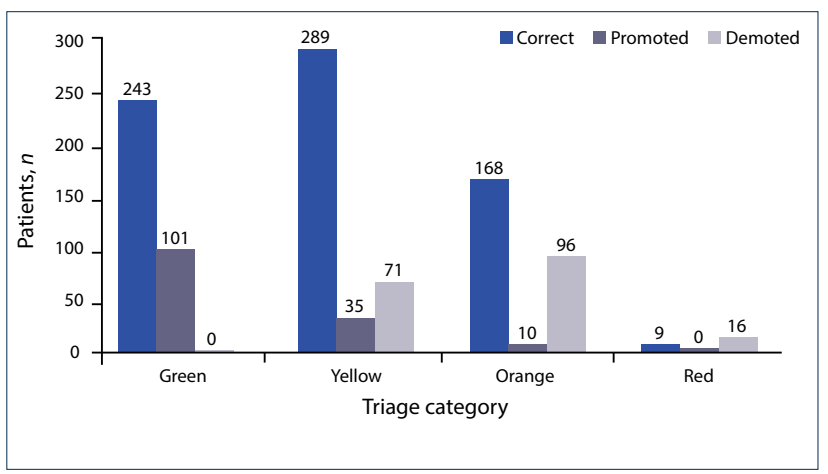

Fig. 1. Numbers of patients who were triaged correctly and incorrectly according to triage category.

The SATS itself does not have performance indicator guidelines on triage accuracy standards to determine whether or not the ED's triage accuracy rate is acceptable.

\section{Trauma v. non-trauma patients}

The majority $(71.0 \%)$ of patients presenting to the ED had nontraumatic pathologies. There was no statistically significant difference in the correct triage rate between trauma and non-trauma patients. However, non-trauma patients were more likely to be incorrectly demoted when incorrectly triaged, whereas trauma patients were more likely to be promoted. This may be related to visual differentiation e.g. a bleeding trauma patient may be interpreted as needing more urgent attention than a patient with chest pain, who may be having a myocardial infarction that cannot be seen with the naked eye.

\section{Promoted and demoted patients}

When looking at promotion, patients in the green category were most commonly promoted - almost one-third (29.4\%) of patients
Table 3. Main reasons for errors made during triage

\begin{tabular}{ll}
\hline Reasons for errors in triage & Errors, $\boldsymbol{n}$ (\%) \\
\hline Discriminator errors & $369(57.8)$ \\
\multicolumn{1}{l}{ Incorrect discriminator usage } & $78 / 369(21.1)$ \\
$\quad$ Failure to record a discriminator & $291 / 369(78.9)$ \\
$\begin{array}{l}\text { Numerical miscalculations (miscalculation } \\
\text { due to addition or transposition error) }\end{array}$ & $137(21.5)$ \\
$\begin{array}{l}\text { Other (incorrect triage placement despite } \\
\text { correct TEWS score, and/or correct }\end{array}$ & $132(20.7)$ \\
$\begin{array}{l}\text { discriminator usage) } \\
\text { Total }\end{array}$ & \\
$\begin{array}{l}\text { *A total of 638 errors were made even though only 329 patients were triaged incorrectly, } \\
\text { i.e. in some cases more than one error was made per patient, and some of the errors also } \\
\text { did not result in an incorrect triage result. }\end{array}$
\end{tabular}

who should have been in this routine category were placed in yellow or above. Of incorrectly demoted patients, one-third of patients in the orange category (very urgent) were placed in the yellow or green categories.

Mis-triaging patients, whether by incorrectly promoting or demoting them, can have detrimental effects. Incorrect promotion increases the number of patients who need to be seen urgently and puts further strain on an already under-resourced and under-staffed system, which may also lead to correctly triaged and more critical patients not receiving treatment within the recommended time. Incorrectly demoting a patient potentially has even more serious consequences. In our study, 82 patients who should have been triaged orange were triaged yellow (Table 2). This means that a patient who should have been evaluated within 10 minutes of arrival in the ED could theoretically have waited for up to an hour, which could be detrimental or even fatal.

Table 4. Mechanisms of errors resulting in a correct or incorrect triage result

\begin{tabular}{lll}
\hline Table 4. Mechanisms of errors resulting in a correct or incorrect triage result \\
\hline Reasons for errors in triage & $\begin{array}{l}\text { Errors resulting in an incorrect } \\
\text { triage result, } \boldsymbol{n} \text { (\%) }\end{array}$ & $\begin{array}{l}\text { Errors nevertheless resulting in a } \\
\text { correct triage result, } \boldsymbol{n} \text { (\%) }\end{array}$ \\
\hline $\begin{array}{l}\text { Discriminator errors } \\
\quad \text { Incorrect discriminator usage }\end{array}$ & $38 / 78(48.7)$ & $40 / 78(51.3)$ \\
$\quad$ Failure to record a discriminator & $133 / 291(45.7)$ & $158 / 291(54.3)$ \\
$\begin{array}{l}\text { Numerical miscalculation (miscalculation due to addition or } \\
\text { transposition error) }\end{array}$ & $68 / 137(49.6)$ & $69 / 137(50.4)$ \\
$\begin{array}{l}\text { Other (incorrect triage placement, despite correct TEWS } \\
\text { score, and/or correct discriminator usage) }\end{array}$ & $132 / 132(100)$ & $0(0)$ \\
Total & $371 / 638(58.2)$ & $267 / 638(41.8)$
\end{tabular}


Table 5. Discriminators most often omitted ${ }^{*}$

\begin{tabular}{lllll}
\hline Discriminator omitted & Total, $\boldsymbol{n}(\mathbf{\%})(\boldsymbol{N = 2 9 1 )}$ & Correctly triaged, $\boldsymbol{n}(\mathbf{\%})^{\dagger}$ & Promotion, $\boldsymbol{n}$ (\%) & Demotion, $\boldsymbol{n}(\%)$ \\
\hline Abdominal pain & $88(30.2)$ & $39(44.3)$ & $2(2.2)$ & $47(53.4)$ \\
Chest pain & $48(16.5)$ & $25(52.1)$ & 0 & $23(47.9)$ \\
Shortness of breath & $44(15.1)$ & $11(25.0)$ & $2(4.5)$ & $31(70.5)$ \\
Vomiting & $22(7.6)$ & $13(59.1)$ & $1(4.5)$ & $8(36.4)$ \\
Reduced level of consciousness & $17(5.8)$ & $13(76.5)$ & 0 & $4(23.5)$ \\
Psychosis/aggression & $17(5.8)$ & $17(100)$ & 0 & 0 \\
$\begin{array}{l}{ }^{*} \text { Pain was the least commonly used discriminator }(0.18 \%) \\
\text { 'Patients who were still correctly triaged despite omission of the correct discriminator. }\end{array}$ & & &
\end{tabular}

Patients in the red category are of the highest priority and therefore at greatest risk of morbidity and mortality if under-triaged. In this study, $16 / 25$ patients $(64.0 \%)$ who should have been triaged red were incorrectly demoted to orange, which is similar to the $66 \%$ undertriage result for the red category found by Dalwai et al..$^{[3]}$ Although we cannot directly compare these results, we can speculate that a similar under-triage result might have been achieved had true acuity levels been determined. When looking at the reasons for mis-triage in the red category, the primary errors were miscalculation and errors in the 'other' category (TEWS were calculated correctly and/ or the correct discriminator was used, but the incorrect category was still chosen).

\section{Reasons for incorrect triaging Discriminator errors}

The main reason for errors in triaging was failure to record a discriminator (Table 3). This refers to circumstances in which patients presented with a problem on the discriminator list, but it was not recorded. Other reasons written by the nurse that were not on the list also constituted an 'incorrect discriminator'. Discriminators allow a patient, regardless of their TEWS score, to be placed immediately into a higher triage category based on the severity of their pathology. Not recording a valid discriminator or using the incorrect discriminator will therefore result in incorrect promotion or demotion.

The leading omitted discriminators were 'abdominal pain', 'chest pain' and 'shortness of breath' (Table 5). Patients with abdominal and chest pain were still correctly triaged half of the time when one of these symptoms was present in the main complaint, even when this were not documented as a discriminator per se. It may be that the discriminator was in fact recognised by the nurse, who triaged the patient appropriately but just did not document it as a discriminator on the triage form itself.

The inter- and intra-reliability of the SATS have been found to have acceptable accuracy. ${ }^{[3]}$ Our findings indicate that certain discriminators may need more explicit guidelines that could improve their utility. It may be especially beneficial to develop such a guideline for the 'pain' discriminator, which was only used twice in the 1091 forms reviewed. This may be due to nurses' lack of confidence in their ability to differentiate between mild, moderate and severe pain, the pain severity discriminator not actually changing the triage category, or, sadly, staff becoming immune to patients' complaints of pain (pain is one of the most common reasons for presentation to the ED). ${ }^{[9]}$

All patients who presented with, or had a history of, psychosis or aggression were triaged orange, even if the patient did not have active 'psychosis or aggression' at the time of presentation to the ED. Triage training should therefore emphasise that a history of psychosis does not necessarily imply current psychosis, and the patient should not be triaged orange on the basis of their psychiatric history alone.

The discriminators most often cited that were not on the SATS list included 'low oxygen saturation, 'increased or decreased blood pressure,' 'chronic chest pain', 'abscess' and 'head injury'. These symptoms or vital signs must have been of concern to the triage nurses, but would have been picked up by the TEWS or other discriminators. The use of 'low oxygen saturation' as a discriminator may have originated from this ED's policy that nurses should 'notify a doctor' if it is present. The use of non-existent discriminators nevertheless resulted in the correct triage result $51.3 \%$ of the time. As the aim of this study was to assess the accuracy rate according to the 2008 SATS guidelines specifically, cases where ED-specific rules were used and/or changed the ultimate triage outcome were regarded as incorrect.

\section{Numerical miscalculations}

Triage errors due to numerical miscalculations occurred because of incorrect addition of the TEWS score even when the correct TEWS column was selected. Miscalculations were also due to incorrect TEWS columns being chosen, resulting in a TEWS score that was an inaccurate representation of the patient's actual condition. Thirty patients had numerical miscalculations that still resulted in the correct triage result, i.e. a TEWS score of 3 still placed the patient correctly in the yellow category, even when the correct TEWS score should have been 4 (also yellow)

\section{Other}

In 132 cases the TEWS score was correct, the correct discriminator was chosen and no incorrect discriminators were used, but the incorrect triage category was still selected. This was the second most common reason for triage errors, yet the explanation for it is uncertain. It appears to be equivalent to correctly calculating a complex mathematical problem in a test and obtaining the correct answer, but transferring an incorrect answer to the answer sheet. The result is wrong, and in the case of patient care may be detrimental.

\section{Study limitations}

In assessing the accuracy of triage performance, the notion of human error is a central contributing factor. All human beings, including healthcare practitioners who are responsible for the triaging of patients, are vulnerable to error. This can include inaccurately recording a patient's physiological vital signs, e.g. blood pressure or respiratory rate, transposing them incorrectly, miscalculating the TEWS, and incorrectly using or not using a discriminator. All these determine the ultimate correctness of the triage outcome. Some errors cannot be accounted for in this study, and evaluating them would require a prospective, observational study. 
As this was a retrospective study making use of the triage forms, it was not possible to evaluate whether the consequences of promotion or demotion mis-triage had an impact on individual patient outcome or the healthcare system and functionality of the ED.

\section{Recommendations}

- Further quality assurance measures for triage need to be instituted. Triage courses or refresher training for all nurses and doctors, not just those involved in triage, should be offered on a continuous basis throughout the year. Training should include the use of case studies (such as validated vignettes) where skills can be practised and timeous feedback delivered. This will increase self-efficacy and, it is hoped, triage accuracy in the ED.

- Quality control measures need to be evaluated regularly. The correct use of the triage tool needs to be monitored by performing 'spot checks' on triage forms to determine whether patients have been triaged correctly.

\section{Conclusions}

Our study showed that patients were correctly triaged $68.3 \%$ of the time. There was no difference in the correct triage rate between trauma and non-trauma patients, but non-trauma patients were more likely to be incorrectly demoted when incorrectly triaged, whereas trauma patients were more likely to be promoted. Incorrect triaging mostly resulted in the promotion of patients who should have been in the green category and demotion of patients who should have been in the orange category. Mis-triaging can be attributed to incorrect or lack of discriminator use, numerical miscalculations and other human errors.
Quality control and quality assurance measures must target training in these areas to minimise mis-triage in the ED.

Disclaimer. The views expressed in the submitted article are those of the authors, and not an official position of the University of the Witwatersrand. Sources of support. No financial support was given for this study, and any costs incurred were covered by the authors.

1. FitzGerald G, Jelinek GA, Scott D, Gerdtz MF. Emergency department triage revisited. Emerg Med 2010;27(2):86-92. http://dx.doi.org/10.1136/emj.2009.077081

2. Scheutz P, Hausfater P, Amin D. Optimizing triage and hospitalization in adult general medical emergency patients: The triage project. BMC Emerg Med 2013;13(12):1-11. http://dx.doi. org/10.1186/1471-227X-13-12

3. Dalwai MK, Twomey M, Maikere J, et al. Reliability and accuracy of the South African Triage Scale when used by nurses in the emergency department of Timergara Hospital, Pakistan. S Afr Med J 2014;104(5):372-375. http://dx.doi.org/10.7196/SAMJ.7604

4. Rosedale K, Smith ZA, Davies H, Wood D. The effectiveness of the South African Triage Score (SATS) 4. Rosedale K, Smith ZA, Davies H, Wood D. The effectiveness of the S
in a rural emergency department. S Afr Med J 2011;101(8):537-540.

5. Twomey M, Wallis LA, Thompson ML, Myers JE. The South African triage scale (adult version) Twomey M, Wallis LA, Thompson ML, Myers JE. The South African triage scale (adult version)
provides valid acuity ratings when used by doctors and enrolled nursing assistants. Afr J Emerg Med provides valid acuity ratings when used by doctors and enroll

6. American College of Surgeons Committee on Trauma. Resources for Optimal Care of the Injured Patient. Chicago: American College of Surgeons, 2014. https://www.facs.org/quality-programs trauma/vrc/resources (accessed 19 October 2016)

7. Burstrom L, Nordberg M, Ornung G, et al. Physician-led team triage based on lean principles may be superior for efficiency and quality? A comparison of three emergency departments with different triage models. Scand J Trauma Resusc Emerg Med 2012;20:57. http://dx.doi.org/10.1186/1757-7241-20-57

8. Molyneux E, Ahmad S, Robertson A. Improved triage and emergency care for children reduce inpatient mortality in a resource-constrained setting. Bull World Health Organ 2006;84(4):314-319. https://www.ncbi.nlm.nih.gov/pmc/articles/PMC2627321/pdf/16628305.pdf (accessed 5 February 2017).

9. Kapoor S, White J, Thorn BE, et al. Patients presenting to the emergency department with acute pain: The significant role of pain catastrophizing and state anxiety. Pain Med 2015;17(6):1069-1078. http://
(a) dx.doi.org $/ 10.1093 / \mathrm{pm} / \mathrm{pnv} 034$

Accepted 25 November 2016. 